\title{
Revision Art. 71 a/b KVV
}

\section{Beat Kipfera , Carsten Witzmann ${ }^{b}$}

${ }^{a}$ Dr. med., Facharzt für Herz- und thorakale Gefässchirurgie, Leiter Vertrauensärztlicher Dienst KPT/CPT, Mitglied FMH; ${ }^{b}$ Rechtsanwalt, EMBA UZH, Leiter Rechtsdienst KPT/CPT

\section{Einleitung}

Mit dem Urteil 9C_334/2010 vom 23.11.2010 (BGE 136 $\mathrm{V}$ 395) hat das Bundesgericht Stellung bezogen zur Kostenübernahme von Medikamenten für seltene Krankheiten ausserhalb der Spezialitätenliste (SL) und der dabei gebotenen Wirtschaftlichkeitsprüfung. Insbesondere äusserten sich die Bundesrichter zu den Voraussetzungen, welche für eine Abgeltung erfüllt sein müssen:

- Die Kosten für ein Arzneimittel ausserhalb der zugelassenen Indikationen respektive der SL-Limitationen können ausnahmsweise übernommen werden, wenn ein Behandlungskomplex vorliegt oder für eine Krankheit, die für die versicherte Person tödlich verlaufen oder schwere und chronische gesundheitliche Probleme nach sich ziehen kann, und wenn wegen fehlender therapeutischer Alternativen keine andere wirksame Behandlungsmethode verfügbar ist; diesfalls muss das Arzneimittel einen hohen therapeutischen (kurativen oder palliativen) Nutzen haben.

\section{Révision de l'art. 71 a/b OAMal}

En 2011, l'OFSP a mis en vigueur une ordonnance (art. $71 \mathrm{a} / \mathrm{b}$ OAMal) qui règle le remboursement de médicaments admis ou non dans la liste des spécialités (LS) et utilisés pour d'autres indications que celles autorisées ou prévues par la limitation.

Suite à des critiques lors de l'application et à une évaluation du «Büro Vatter", I'OFSP a été contraint de réviser ces dispositions. Nous souhaitons ci-après nous prononcer à ce sujet:

L'art. 28 al. 3 let. g introduit une obligation de transmettre sans but précis, ce qui est à notre sens illicite.

A I'art. 71a OAMal, une limite de prix de 90\% est introduite, ce qui n'est pas envisageable pour plusieurs raisons.

La possibilité pour l'assureur de recourir contre un refus de prix du titulaire de l'autorisation continue de faire défaut, tout comme l'obligation de livrer. Des questions demeurent également concernant la règlementation relative au prix de référence et à l'importation de médicaments (art. 71b et art. 71c). Le nouvel art. $71 \mathrm{~d}$ est contraire au système et doit donc être rejeté.

S'il est nécessaire de réviser l'art. 71 a/b OAMal, la direction prise n'est cependant pas la bonne!

- Für die Zulassung eines Off-Label-Use kann aber nicht jeglicher therapeutische Nutzen genügen, könnte doch sonst in jedem Einzelfall die Beurteilung des Nutzens an die Stelle des gesetzlichen Listensystems treten und dieses unterwandern.

- Da das gesetzliche System auch der Wirtschaftlichkeit dient, muss insbesondere vermieden werden, dass durch eine extensive Praxis der ordentliche Weg der Listenaufnahme durch Einzelfallbeurteilungen ersetzt und dadurch die mit der Spezialitätenliste verbundene Wirtschaftlichkeitskontrolle umgangen wird (alle Erw. 5.2).

Im Nachgang $\mathrm{zu}$ diesen wegweisenden Erwägungen setzte das Bundesamt für Gesundheit (BAG) per 1. März 2011 Art. 71a/b KVV ${ }^{1}$ in Kraft und regelte damit die Vergütung von Arzneimitteln auf oder ausserhalb der SL bei Anwendung ausserhalb der Indikation und Limitation auf Verordnungsstufe.

Von Beginn weg war die Umsetzung von Art. 71a/b KVV aus zwei Gründen schwierig:

- Die Nutzenbewertung durch die Vertrauensärzte der Krankenversicherer (KV) konnte sich zu Beginn auf keine Erfahrungswerte und anerkannte Kriterien stützen. Iterativ wurden deshalb solche Instrumente entwickelt, die heute bei der Beurteilung von allen Stakeholdern verwendet werden.

- Die Preisfestsetzung durch die KV ist für die Zulassungsinhaber (ZI) nicht verpflichtend. Wohl kann der Versicherer die Höhe der Abgeltung entsprechend der Nutzenbeurteilung festlegen, der ZI kann jedoch nicht gezwungen werden, sein Produkt für diesen Preis zu liefern. Entgegen den Bestimmungen des KVG (Tarifschutz, Art. 44 KVG) kann sich somit für den Patienten die Situation ergeben, dass es bei einer Pflichtleistung im Grundversicherungsbereich zu ungedeckten Kosten kommen kann.

Im Nachgang zu einer Evaluation des "Büro Vatter» ${ }^{2}$ hat das BAG die Art. 71a/b KVV überarbeitet und diesen Entwurf nun in die Vernehmlassung geschickt. Im Folgenden möchten wir die vorgeschlagenen Änderungen aus unserer Sicht diskutieren.

Eine Gegenüberstellung der aktuell gültigen und der auf 1. Januar 2017 vorgesehenen Fassung ist online abrufbar (siehe Fussnote 1). 


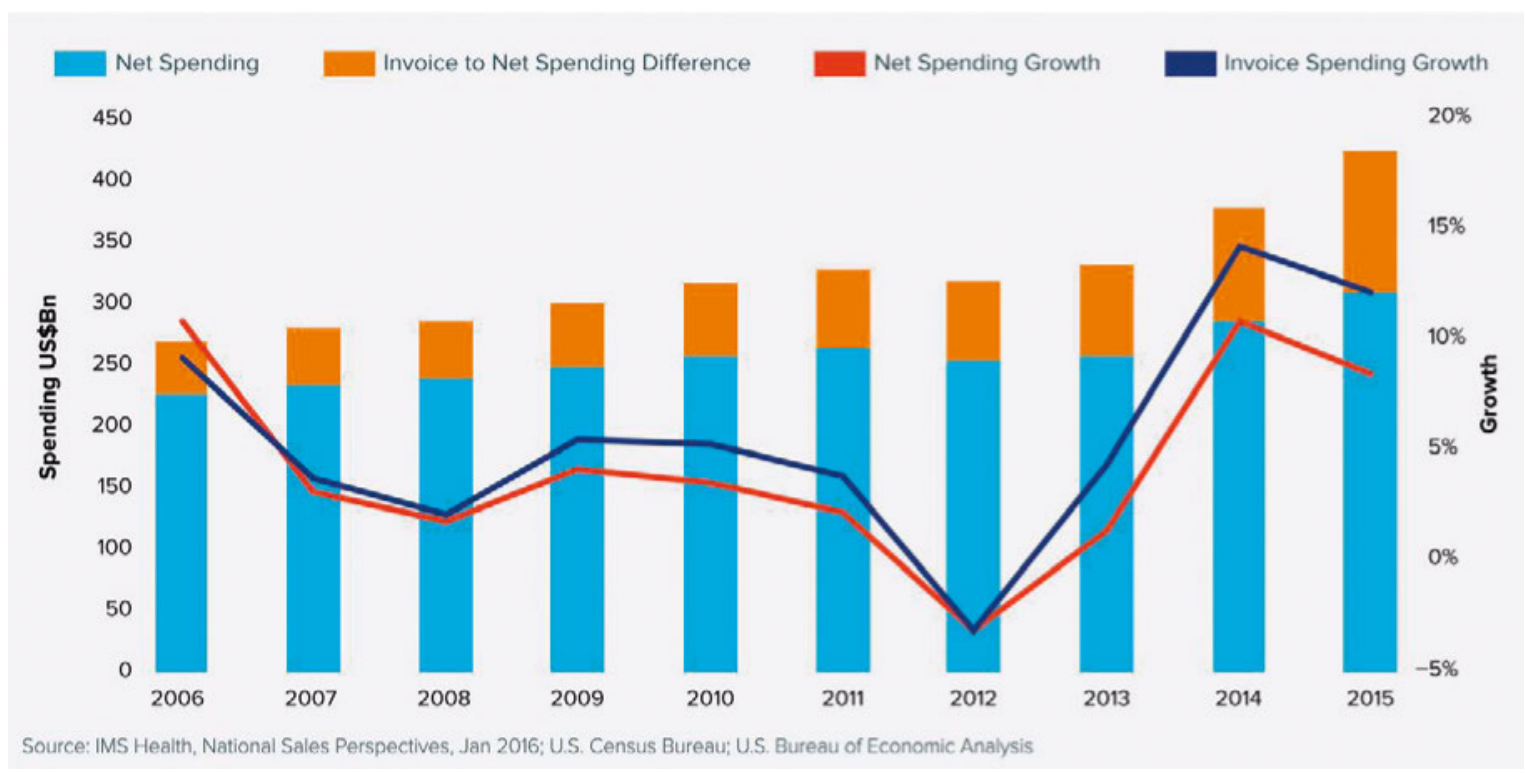

Abbildung 1: Gesamte Ausgaben für Medikamente, USA 2006-2015.

\section{Würdigung}

Neu Art. 28 Abs. 3 Bst. g KVV Big Data ohne Plan

Die Verwaltung hat ihr Handeln nach dem Gebot der Verhältnismässigkeit auszurichten, das Eingreifen des Staates sollte auf das Notwendige beschränkt sein.

Diese Vorgabe ist mit Art. 28 Abs. 3 Bst. g KVV unserer Ansicht nach verletzt:

\section{- Datenlieferung:}

- Es wird eine Datenbeschaffung ohne klare Zweckbindung und Auftrag implementiert.

- Es sei daran erinnert, dass eine «Datensammlung auf Vorrat» unzulässig ist (Art. 4 Abs. 2 DSG).

- Eine Datensammlung ohne vorher festgelegte Auswertungsparameter ermöglicht eine missverständliche Interpretation.

- Das BAG hat die Gelegenheit verpasst, die zunehmende Off-Label-Verwendung (OLU) von innovativen Medikamenten systematisch zu erfassen, wie dies in anderen Ländern umgesetzt ist [1].

\section{- Höhe der Vergütung}

- Zwischen KV und ZI bestehen Verträge über die Preise für Medikamente, welche unter den Bedingungen von Art 71a/b KVV verwendet werden. Diese Verträge sind legitim und geben Rechtssicherheit in einem durch das KVG nicht abgedeckten Rechtsverhältnis (ZI sind keine Leistungserbringer im KVG).

- Zwingt man die KV, die Höhe der Vergütung offenzulegen, wird die vertraglich festgelegte Ver- traulichkeit über den Vertragsinhalt verletzt. Eine solche Bekanntgabe ist kartellrechtlich problematisch, da gestützt auf das Öffentlichkeitsprinzip von Mitbewerbern eine Veröffentlichung dieser Daten verlangt werden kann.

\section{Neu Art. 71a KVV - Regression toward the mean}

Der rev. Art. 71a KVV regelt die Übernahme der Kosten eines Arzneimittels der SL ausserhalb der genehmigten Fachinformation oder Limitierung.

Die Änderung bezüglich Art. 71a KVV sieht zwei gewichtige Veränderungen vor: die Festlegung einer Obergrenze von $90 \%$ des Fabrikabgabepreises (FAP) der SL als Höchstpreis und die Festsetzung des FAP als Richtpreis. Beide Änderungen sind für uns nicht sinnvoll:

\section{- Preisobergrenze von 90\%}

- Die bisherige Rabattierung korreliert mit der Nutzenbewertung durch die Vertrauensärzte: Ein Hoher Nutzen ("A») wird mit einer kleineren Rabattierung als ein Mässiger Nutzen («B») versehen, wogegen ein Potentieller Nutzen ( $(\mathrm{C} »)$ keine unmittelbare Abgeltung durch die KV auslöst, sondern eine initiale Kostenübernahme durch den ZI mit nachfolgender gemeinsamer Beurteilung des Therapieerfolgs (pay for perfomance).

- Die Einführung einer Preisobergrenze von 90\% wird die bisherige Rabattierung dahingehend verändern, als dass der neu eingeführte, absolute Wert als Referenzpunkt für die zukünftige Rabattierung dienen wird. Das heisst, dass in den meisten Fällen die Rabatte kleiner ausfallen werden als heute. 
- Wir meinen, diese Festsetzung einer Höchstgrenze mit einem absoluten Wert ist auch kartellrechtlich problematisch, da das BAG damit indirekt eine Preisabsprache "verordnet».

\section{- Fehlende Eskalation}

- Die neu vorgeschlagene Sprachregelung («Nach Absprache mit dem Zulassungsinhaber bestimmt er [der Versicherer] die Höhe der Abgeltung») setzt unserer Meinung nach explizit eine Einigung zwischen KV und ZI vor der Festlegung des Preises voraus. Die Formulierung ist auch dahingehend unscharf, dass nicht deutlicher ausgedrückt wird, dass die Hoheit der Preisfestlegung beim KV liegt.

- Es wäre sachdienlicher gewesen, folgenden neuen Abs. 3 einzufügen: «Kann keine Absprache über die Höhe der Vergütung erzielt werden, so geht der Versicherer in Vorleistung und beschreitet unter Abtretung der Ansprüche durch den Versicherten den bestehenden Rechtsweg.»

- Fehlende Lieferpflicht des Zulassungsinhabers

- Wir sind der Meinung, dass es der Verordnungsgeber unterlassen hat, die Lieferpflicht des ZI klar zu regeln.

- Er könnte dies problemlos durch eine Ergänzung in Art. $65 \mathrm{KVV}$ anbringen, welche den Zulassungsinhaber verpflichten würde, bei Registrierung oder Aufnahme bzw. Einreichung eines Gesuchs um Aufnahme eines Medikamentes in die SL die Preisfestsetzung nach Art. 71a-d KVV anzuerkennen, vorausgesetzt, diese ist nicht willkürlich.

\section{- Fabrikabgabepreis}

Bei den ZI wie auch bei den KV würde die Einführung des FAP einen zusätzlichen Aufwand generieren, da die heutigen ERP-Systeme auf dem Publikumspreis basieren.

\section{Neu Art. 71b KVV - Mein Freund Google ${ }^{\circledR}$}

Art. 71b KVV regelt die Übernahme der Kosten eines vom Institut zugelassenen und nicht in die SL aufgenommenen Arzneimittels.

Folgende geplante Revisionen sind unserer Meinung nach abzuändern:

\section{- Referenzpreise im Ausland}

- Referenzpreise im Ausland sind oft «Schaufenster-Preise» und widerspiegeln nicht den wahren Preis, den der Leistungserbringer (Resp. KV) bezahlen muss. Oft bestehen auch im selben Land unterschiedliche Verkaufspreise, wie das Beispiel der USA anschaulich demonstriert:

- Medicare und Medicaid, die staatlichen Sozialversicherungen, sind gesetzlich verpflichtet, den Herstellerpreis zu bezahlen.

- Die privaten Versicherungen (wie Aetna, United Health uam.) können die Preise verhandeln, resp. sie kaufen die Medikamente über Grosshändler ein, welche markante Rabatte durchsetzen können (siehe Abb. 1) [2].

- Ein aktuelles Beispiel stellt dieses Problem dar (siehe Tab. 1).

- Sachdienlicher wäre für uns eine Ausführungsbestimmung zur Preisübernahme durch den ZI und eine Verpflichtung zur automatischen Nennung der weltweiten Referenzpreise.

\section{Neu Art. 71c KVV - Der Versicherer als Makler}

In Art. 71c KVV wird versucht, den Import und die nachfolgende Abgeltung von verwendungsfertigen, im Ausland zugelassenen Arzneimitteln zu regeln, welche weder registriert sind noch eine SL-Listung haben.

Diese Bestimmungen werfen einige Fragen auf:

- Was wird als "gleichwertig anerkanntes Zulassungssystem» akzeptiert?

- Wie organisiert der Leistungserbringer einen Parallelimport ohne Intermediär?

- Wie verhält sich diese Bestimmung zum Verbot des Parallelimports von preisregulierten, patentgeschützten Arzneimitteln?

Diese Fragen bleiben unbeantwortet.

\section{Neu Art. 71d KVV - Moral Hazard 2.0}

In Abs. 4 wird ausgeführt, wie mit einem individuellen Therapieversuch (entspr. der Nutzenbewertung "C») verfahren werden soll.

Diese Änderung in der Verordnung ist nicht akzeptabel und muss entfernt werden:

- Die Nutzenbewertung im Nachgang zu einem Therapieversuch hebelt die Nutzenbewertung durch den Vertrauensarzt aus, wie sie für eine Kostenübernahme unter Art. 71a KVV gefordert wird. Mithin werden Kosten ohne Beachtung der WZW-Kriterien (Wirksamkeit, Zweckmässigkeit, Wirtschaftlichkeit) durch die Krankenpflegeversicherung übernommen.

- Eine kritische Würdigung einer Therapieoption durch den Vertrauensarzt wird verhindert. Dies ist umso bedauerlicher, als immer mehr Medikamente im Rahmen von «accelerated approvals» mit marginaler Datenlage zugelassen werden:

Meist sind nur Phase-I- oder Phase-II-Studien notwendig für die Zulassung in den USA. Die Effekte 
Tabelle 1: Beispiel Osimertinib (generic name).

\begin{tabular}{|c|c|c|}
\hline Land & Preis & Bemerkung \\
\hline USA & $\begin{array}{l}12600 \text { USD } \\
\text { (12239 CHF) }\end{array}$ & Therapiekosten pro Monat bei $80 \mathrm{mg}$ pro Tag \\
\hline United Kingdom & $\begin{array}{l}4722,30 \mathrm{GBP} \\
\text { (5953 CHF) }\end{array}$ & $\begin{array}{l}\text { Therapiekosten pro Monat bei } 80 \mathrm{mg} \text { und } 40 \mathrm{mg} \text { pro Tag } \\
\text { "The company has agreed a patient access scheme with the Department of Health. } \\
\text { If osimertinib had been recommended, this scheme would provide as simple } \\
\text { discount to the list price of osimertinib with the discount applied at the point } \\
\text { of purchase or invoice. The level of the discount is commercial in confidence.» }\end{array}$ \\
\hline Herstellerangebot an KPT & $9500 \mathrm{CHF}$ & $\begin{array}{l}\text { Begründung der Zulassungsinhaberin: Bisher haben wir alle Vereinbarungen } \\
\text { mit Krankenkassen auf Basis FAP von } 9500 \mathrm{CHF} \text { abgeschlossen. Im Sinne der } \\
\text { Gleichbehandlung können wir nicht für jede einzelne Krankenkasse einen unter- } \\
\text { schiedlichen FAP ansetzen. } \\
\text { (Anmerkung: } 9500 \mathrm{CHF} \text { ist der beim BAG eingereichte Preis.) }\end{array}$ \\
\hline
\end{tabular}

werden mit Surrogat-Markern bewertet, oft wird als Komparator entweder Placebo oder BSC (best supportive care) verwendet (siehe Tab.1) [3].

Die wenigsten so zugelassenen Medikamente erfüllen die Bedingungen an einen "clinically meaningful outcome», wie sie von der American Society of Clinical Oncology [4] gefordert werden.

\section{Fazit}

Das BAG hat es verpasst, die bekannten Probleme von Art. 71a/b KVV zu lösen:

- Die Einbindung der ZI ins KVG wurde nicht umgesetzt: Die ZI sind noch immer nicht verpflichtet, Preisfestsetzungen der Versicherer anzuerkennen.

- Die Problematik des Nutzens von neuen Medikamenten, welche überwiegend mit Hilfe von Surrogat-Endpunkten zugelassen wurden, hat man akzentuiert.

Weiter ist festzustellen, dass den Empfehlungen der ständerätlichen Kommission für soziale Sicherheit und Gesundheit vom 12.2.2014 zur Motion Steitert (12.3816) nicht in allen Punkten gefolgt worden ist: So wird nicht klar verhindert, dass "Patienten einen Teil der Kosten übernehmen müssen, weil die Herstellerfirma einen höheren Preis für ein Arzneimittel verlangt, als die Krankenkasse als wirtschaftlich beur- teilt». Es hätte unseres Erachtens nichts dagegen gesprochen, den Tarifschutz (Art. $44 \mathrm{KVG}$ ) in die neuen Bestimmungen zu integrieren.

Die Verordnungsänderung zeigt weiter eindrücklich, wie sich das BAG um eine entscheidende gesellschaftliche Frage drückt, zu deren Beantwortung das Bundesgericht im eingangs erwähnten Entscheid BGE 136 V 395 aufgefordert hat:

- Welche Kosten und Leistungen kann und soll unser Sozialversicherungssystem noch finanzieren?

- Wo liegt die Grenze?

Es wäre nun an der Zeit, statt die Kraft für eine fragwürdige Änderung einer Verordnung zu verschwenden, endlich den politischen und gesellschaftlichen Dialog über den Begriff «value» zu initiieren. Wir werden dieser Diskussion nicht ausweichen können!

\section{Bildnachweise}

(C) QuintilesIMS

Literatur

1 Emmerich J, Dumarcet N, Lorence A. France's New Framework for Regulating Off-Label Drug Use. New England Journal of Medicine, 2012;367(14):1279-81.

2 IMS, Medicines Use and Spending in the U.S. A Review of 2015 and Outlook to 2020. IMS Health Care, 2016.

3 Eaton KD, Jagels B, Martins RG. Value-Based Care in Lung Cancer. The Oncologist, 2016;21(8):903-6.

4 Ellis LM, et al. American Society of Clinical Oncology Perspective: Raising the Bar for Clinical Trials by Defining Clinically Meaningful Outcomes. Journal of Clinical Oncology, 2014;32(12):1277-80. 
Aktuelle Version Art 71 KVV (07.2016)

Art 71a Übernahme der Kosten eines Arzneimittels der Spezialitätenliste ausserhalb der genehmigten Fachinformation oder Limitierung

1 Die obligatorische Krankenpflegeversicherung übernimmt die Kosten eines in die Spezialitätenliste aufgenommenen Arzneimittels für eine Anwendung ausserhalb der vom Institut genehmigten Fachinformation oder ausserhalb der in der Spezialitätenlistefestgelegten Limitierung nach Artikel 73, wenn:

a. der Einsatz des Arzneimittels eine unerlässliche Voraussetzung für die Durchführung einer anderen von der obligatorischen Krankenpflegeversicherung übernommenen Leistung bildet und diese eindeutig im Vordergrund steht; oder

b. vom Einsatz des Arzneimittels ein grosser therapeutischer Nutzen gegen eine Krankheit erwartet wird, die für die versicherte Person tödlich verlaufen oder schwere und chronische gesundheitliche Beeinträchtigungen nach sich ziehen kann, und wegen fehlender therapeutischer Alternativen keine andere wirksame und zugelassene Behandlungsmethode verfügbar ist.

Sie Übernimmt die Kosten des Arzneimittels nur auf besondere Gutsprache des Versicherers nach vorgängiger Konsultation des Vertrauensarztes oder der Vertrauensärztin

3 Die zu übernehmenden Kosten müssen in einem angemessenen Verhăltnis zum therapeutischen Nutzen stehen. Der Versicherer bestimmt die Höhe der Vergütung. Der in der Spezialitätenliste aufgeführte Preis gilt als Höchstpreis.

\section{Art. 71b Öbernahme der Kosten eines nicht in die Spezialitätenlist aufgenommenen Arzneimittels}

1 Die obligatorische Krankenpflegeversicherung übernimmt die Kosten eines vom Institut zugelassenen verwendungsfertigen Arzneimittels, das nicht in die Spezialitätenliste aufgenommen ist, für eine Anwendung innerhalb oder ausserhalb der Fachinformation, wenn die Voraussetzungen nach Artikel 71a Absatz 1 Buchstabe a oder b erfült sind.

2 Sie übernimmt die Kosten eines vom Institut nicht zugelassenen Arzneimittels, das nach dem Heilmittelgesetz eingeführt werden darf, wenn die Voraussetzungen nach Artikel $71 a$ Absatz 1 Buchstabe a oder b erfüllt sind und das Arzneimittel von einem Land mit einem vom Institut als gleichwertig anerkannten Zulassungssystem für die entsprechende Indikation zugelassen ist.

3 Sie übernimmt die Kosten des Arzneimittels nur auf besondere Gutsprache des Versicherers nach vorgängiger Konsultation des Vertrauensarztes oder der Vertrauensärztin.

4 Die zu übernehmenden Kosten müssen in einem angemessenen Verhältnis zum therapeutischen Nutzen stehen. Der Versicherer bestimmt die Höhe der Vergütung.
Version Art $71 \mathrm{KVV}$ anhand Vernehmlassung BAG

\section{Art. 28 Abs. 3 Bst. g}

3 Die Versicherer müssen dem BAG jährlich pro versicherte Personen namentlich folgende Daten weitergeben:

g. bei einer Vergútung nach den Artikeln $71 a-71 c$ das Eingangsdatum des Gesuchs um Kostengutsprache, die Indikation, den Namen des Arzneimittels, den Namen der Zulassungsinhaberin, das Datum des Leistungsentscheids sowie die Hōhe der Vergütung.

Gliederungsartikel vor Art. 71

4a. Abschnitt: Vergütung von Arzneimitteln im Einzelfall

Art 71a Übernahme der Kosten eines Arzneimittels der Spezialitätenliste ausserhalb der genehmigten Fachinformation oder Limitierung

1 Die obligatorische Krankenpflegeversicherung übernimmt die Kosten eines in die Spezialitätenliste aufgenommenen Arzneimittels für eine Anwendung ausserhalb der vom Institut genehmigten Fachinformation oder ausserhalb der in der Spezialitätenlistefestgelegten Limitierung nach Artikel 73, wenn:

a. der Einsatz des Arzneimittels eine unerlässliche Voraussetzung für die Durchführung einer anderen von der obligatorischen Krankenpflegeversicherung übernommenen Leistung bildet und diese Krankenpflegeversicherung Ubernommenen Leistung bildet und diese b. vom Einsatz des Arzneimittels ein grosser therapeutischer Nutzen gegen eine Krankheit erwartet wird, die für die versicherte Person tödlich verlaufen oder schwere und chronische gesundheitliche Beeinträchtigungen nach sich ziehen kann, und wegen fehlender therapeutischer Alternativen keine andere wirksame und zugelassene Behandlungsmethode verfügbar ist.

2 Der Versicherer vergütet höchstens 90 Prozent des Fabrikabgabepreises der Spezialitätenliste. Nach Absprache mit der Zulassungsinhaberin bestimmt er die Höhe der Vergūtung.

Art. 71b Öbernahme der Kosten eines vom Institut zugelassenen nicht in die Spezialitätenliste aufgenommenen Arzneimittels

1 Die obligatorische Krankenpflegeversicherung übernimmt die Kosten eines vom Institut zugelassenen verwendungsfertigen Arzneimittels, das nicht in die Spezialitätenliste aufgenommen ist, für eine Anwendung innerhalb oder ausserhalb der Fachinformation, wenn die Voraussetzungen nach Artikel $71 a$ Absatz 1 Buchstabe a oder b erfült sind.

2 Die Wirtschaftlichkeit wird aufgrund eines Auslandpreisvergleichs nach Artikel $65 b$ Absatz 2 Buchstabe a beurteilt. Für die Ermittlung des durchschnittlichen Fabrikabgabepreises der Referenzländer ist Artikel $65 b$ Absătze 3 und 4 massgebend.

3 Die Zulassungsinhaberin gibt dem Versicherer auf Anfrage die zum Zeitpunkt des Gesuchs um Kostenübernahme geltenden Fabrikabgabepreise der Referenzländer bekannt.

4 Der Versicherer vergütet höchstens 90 Prozent des durchschnittlichen Fabrikabgabepreises der Referenzländer. Nach Absprache mit der Zulassungsinhaberin bestimmt er die Höhe der Vergütung.

Art. 71c Öbernahme der Kosten eines vom Institut nicht zugelassenen importierten Arzneimittels

1 Die obligatorische Krankenpflegeversicherung übernimmt die Kosten eines vom Institut nicht zugelassenen verwendungsfertigen Arzneimittels, das nach dem Heilmittelgesetz eingeführt werden darf, sofern die Voraussetzungen nach Artikel $71 a$ Absatz 1 Buchstabe a oder b erfüllt sind und das Araneimittel von einem Land mit einem vom Institut als gleichwertig anerkannten Zulassungssystem für die entsprechende Indikation zugelassen ist.

2 Der Versicherer vergütet die Kosten, zu denen das Arzneimittel aus dem Ausland importiert wird. Der Leistungserbringer achtet bei der Auswahl des Landes, aus dem 
er das Arzneimittel importiert, auf die Kosten. Wird das Arzneimittel in anderen Lăndern vertrieben, in denen es zu einem günstigeren Preis angeboten wird, so kann der Versicherer verlangen, dass der Leistungserbringer das Arzneimittel aus einem dieser Länder importiert.

\section{Art. 71d Gemeinsame Bestimmungen}

1 Die obligatorische Krankenpflegeversicherung übernimmt die Kosten des Arzneimittels nur auf besondere Gutsprache des Versicherers nach vorgăngiger Konsultation des Vertrauensarztes oder der Vertrauensärztin.

2 Der Versicherer überprüft, ob die von der obligatorischen Krankenpflegeversicherung übernommenen Kosten in einem angemessenen Verhältnis zum therapeutischen Nutzen stehen.

3 Ist das Gesuch um Kostengutsprache vollständig, so entscheidet der Versicherer innert zwei Wochen darüber.

4 Kann bei einem im Rahmen eines individuellen Therapieversuchs eingesetzten Arzneimittel ein grosser Nutzen erst nach dem Entscheid ūber die Kostengutsprache festgestellt werden, so bestimmt der Versicherer auf Vorschlag des Leistungserbringers und der Zulassungsinhaberin vor Beginn der Therapie, wann geprüft wird, ob ein grosser Nutzen besteht. Die Dauer der Vergütung des Arzneimittels im Rahmen des Therapieversuchs beträgt in der Regel höchstens 12 Wochen. Ist ausnahmeweise ein längerer Therapieversuch für die Bestimmung des grossen Nutzens erforderlich, so hat der Leistungserbringer dies im Gesuch um Kostengutsprache zu begründen.

5 Der Höchstpreis eines in der Schweiz erhältlichen Arzneimittels setzt sich aus dem nach Artikel $71 a$ Absatz 2 oder $71 b$ Absatz 4 vom Versicherer vergüteten Fabrikabgabepreis, dem Vertriebsanteil nach Artikel 67 Absatz 1quater und der reduzierten Mehrwertsteuer zusammen. Bei einem importierten Arzneimittel werden die effektiven Vertriebskosten vergütet. 